\title{
CONTRIBUTION À L'APPROCHE MORPHO-STRATIGRAPHIQUE DU PLÉISTOCENE SUPÉRIEUR DU NORD DE LA FRANCE : COLMATAGE D'UN VALLON SEC DE LA GOHELLE (AVION, PAS-DE-CALAIS)
}

Deux transects réalisés à travers un vallon sec élémentaire de la Gohelle (pays crayeux entre Artois et plaines du Nord) montrent la conservation locale, en position d'interfluve ou en partie supérieure du versant, d'un complexe de sols depuis l'Eemien jusqu'au Pléniglaciaire moyen weichselien. Un remaniement de ce complexe avec mélange de craie témoigne d'une forte érosion ultérieure du versant. De même, une formation fluviatile grossière (graviers de craie) témoigne d'une phase d'activité du réseau de vallées, probablement à la transition Pléniglaciaire moyen/supérieur. Le lœss du Pléniglaciaire supérieur, déposé sur le versant sous le vent sur plus de 7 mètres d'épaisseur, scelle l'ensemble des unités antérieures et remblaye un paléo-vallon (nettement plus bas que le talweg actuel). La dissymétrie modérée du profil transversal ne permettait pas de présumer, avant l'opération de sondages, de l'ampleur de l'accumulation éolienne.

Mots-clés : vallon sec, vallée dissymétrique, Weichselien, accumulation lœssique, Gohelle.

\section{ABSTRACT}

CONTRIBUTION TO THE MORPHO-STRATIGRAPHIC APPROACH OF THE UPPER PLEISTOCENE IN THE NORTHERN FRANCE: FILLING UP OF A DRY VALLEY IN THE GOHELLE COUNTRY (AVION, PAS-DE-CALAIS)

Two cross-sections through a small dry valley of the Gohelle (chalky country between Artois and lowlands of Northern France) show the preservation in places of a soils complex from the Eemian up to the Weichselian Middle Pleniglacial, upon an interfluve or the upper part of the slope. A reworking of the soils complex shows a subsequent strong slope erosion. In a same way, a coarse fluvial formation (chalk gravels) shows an active phase of the valleys network, probably at the transition between Middle and Upper Pleniglacial. The upper pleniglacial lœss is settled over more 7 meters on the leeward slope and filled up a palaeovalley (clearly lower than the current talweg), overlying the previous stratigraphic units. Before the deep test pits campaign, the slight dissymmetrical transverse profile didn't allow assuming the great aeolian deposits extent.

Key-words: dry valley, dissymmetric valley, Weichselian, lœssic deposition, Gohelle country.

\section{1 - LOCALISATION, CONTEXTE ET MÉTHODOLOGIE}

Une série de sondages profonds en puits a été réalisée à la pelle mécanique au sud de la ville d'Avion, sur prescription du Service Régional de l'Archéologie du Nord-Pas-de-Calais dans le cadre d'un diagnostic archéologique préventif (recherche d'un éventuel gisement paléolithique) (Deschodt in Lefèvre, 2006). La zone sondée se situe dans la Gohelle, pays crayeux formant un glacis entre le talus bordier de l'Artois et le bas-pays du Nord de la France (fig. 1). Le réseau hydrographique est très lâche, constitué de petites rivières dont la plus importante est la Souchez. Celle-ci prend sa source dans l'Artois, traverse la Gohelle et constitue l'extrémité amont du bassin de la Deûle. La région se caractérise par le développement, au pied du plateau artésien, d'un réseau de petits vallons secs. La couverture lœssique est discontinue avec des affleurements crayeux.

Nos observations ont permis de réaliser deux transects perpendiculaires à un de ces vallons secs élémentaires, vers 50 mètres d'altitude ( $\mathrm{n}^{\circ} 2$ sur la figure 1 ), distant d'un peu plus d' $1 \mathrm{~km}$ des observations de l'ancienne briqueterie de Vimy, en position d'interfluve vers $66 \mathrm{~m}$ d'altitude ( ${ }^{\circ} 1$ sur la figure 1$)$.

Le front d'exploitation de l'ancienne briqueterie, haut d'environ 5 mètres, a été partiellement levé (Sommé, 1975 ; Sommé \& Tuffreau, 1976). La coupe (fig. 2) montre une couverture lœssique régulière, épaisse 
d'environ 4 mètres (1 à 3). Sa base est constituée de limon lité à petites fentes de gel (3) qui recouvre un important hiatus stratigraphique (4) ponctué de grandes fentes de gel (fines, profondes d'environ $1 \mathrm{~m}$ et
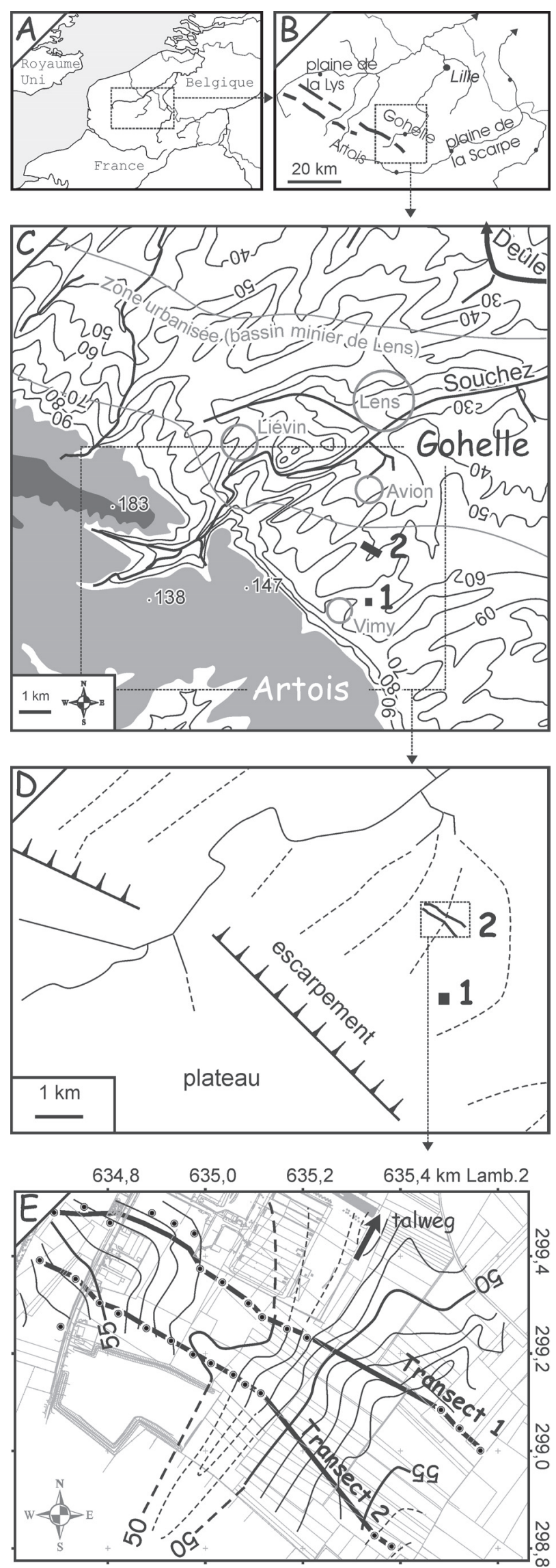

localement denses). Ce niveau érosif plan et régulier tronque l'ensemble des unités sédimentaires sousjacentes : sables et limons lités (5) en ravines peu profondes (inférieures au mètre) et larges (plusieurs mètres) qui affectent le « sol de Warneton » (5), ce dernier préservé sur quelques mètres au sommet du « sol de Rocourt » (6). Les formations antérieures (7 à 10), entraperçues, témoignent d'un bilan sédimentaire complexe pour le Pléistocène moyen.

\section{2 - DESCRIPTION ET SYNTHÈSE LITHO-STRATIGRAPHIQUE}

A Avion, nous synthétisons l'ensemble des terrains rencontrés au-dessus du substrat crayeux en 12 grandes unités stratigraphiques (fig. 3).

\section{Unité 1 : Labours}

La zone sondée est constituée de champs labourés, avec en surface un horizon de limon humifère sombre d'environ 0,3 m d'épaisseur (Ap).

\section{Unité 2 : Colluvions récentes}

Sous le labour, deux zones de colluvions ont été observées. La première, dans le talweg, est constituée d'un limon brun à gravier de craie abondant. D'épaisseur métrique, il repose directement sur la craie fragmentée.

La seconde zone de colluvions, avec une base à environ $0,6 \mathrm{~m}$ de profondeur, est moins épaisse mais se développe largement sur le versant exposé à l'est du transect 2. Ce limon brun issu du remaniement de l'unité 3 comporte quelques éclats métalliques et des surcreusements dus à des trous d'obus. Dans ce cas, les colluvions résultent d'un labourage par les bombardements de la Première Guerre mondiale puis d'un nivellement anthropique lors de la remise en état des terrains.

\section{Unité 3 : Horizon Bt du sol de surface}

Le sol brun lessivé actuel (limon brun argileux formant des structures prismatiques décimétriques), se développe sur le lœss sous-jacent et est d'épaisseur très variable. Il dépasse localement $2 \mathrm{~m}$ de profondeur mais se réduit en général à environ 1 mètre d'épaisseur.

Fig. 1 - Localisation

$A$ et $B$ / cartouches de localisation d'après P.-G. Salvador (université, Lille 1) ; C/ topographie, d'après la carte IGN 1/100000 (mètres Lallemand) ; gris $>100$ mètres ; gris foncé $>150$ mètres ; $D /$ croquis du réseau de talweg ; $\mathrm{E} /$ localisation des sondages, topographie en mètres IGN 69, d'après des points cotés du plan d'aménagement et les levés archéologiques ; 1/ briqueterie de Vimy (Sommé, 1975) ; 2/ zone sondée, transects

Fig. 1 - Location

A and B/frame according to P.-G. Salvador (université, Lille 1) ; C/ topography, according to the 1/100000 IGN map (Lallemand survey); grey > 100 meters; dark grey > 150 meters: D/ talwegs network sketch; E/ Deep test pits location and topography (IGN 69), according to the spots height on the planning map and the archaeological survey; 1/Vimy's brickyard (Sommé, 1975) ; 2/ bored area, cross-sections 


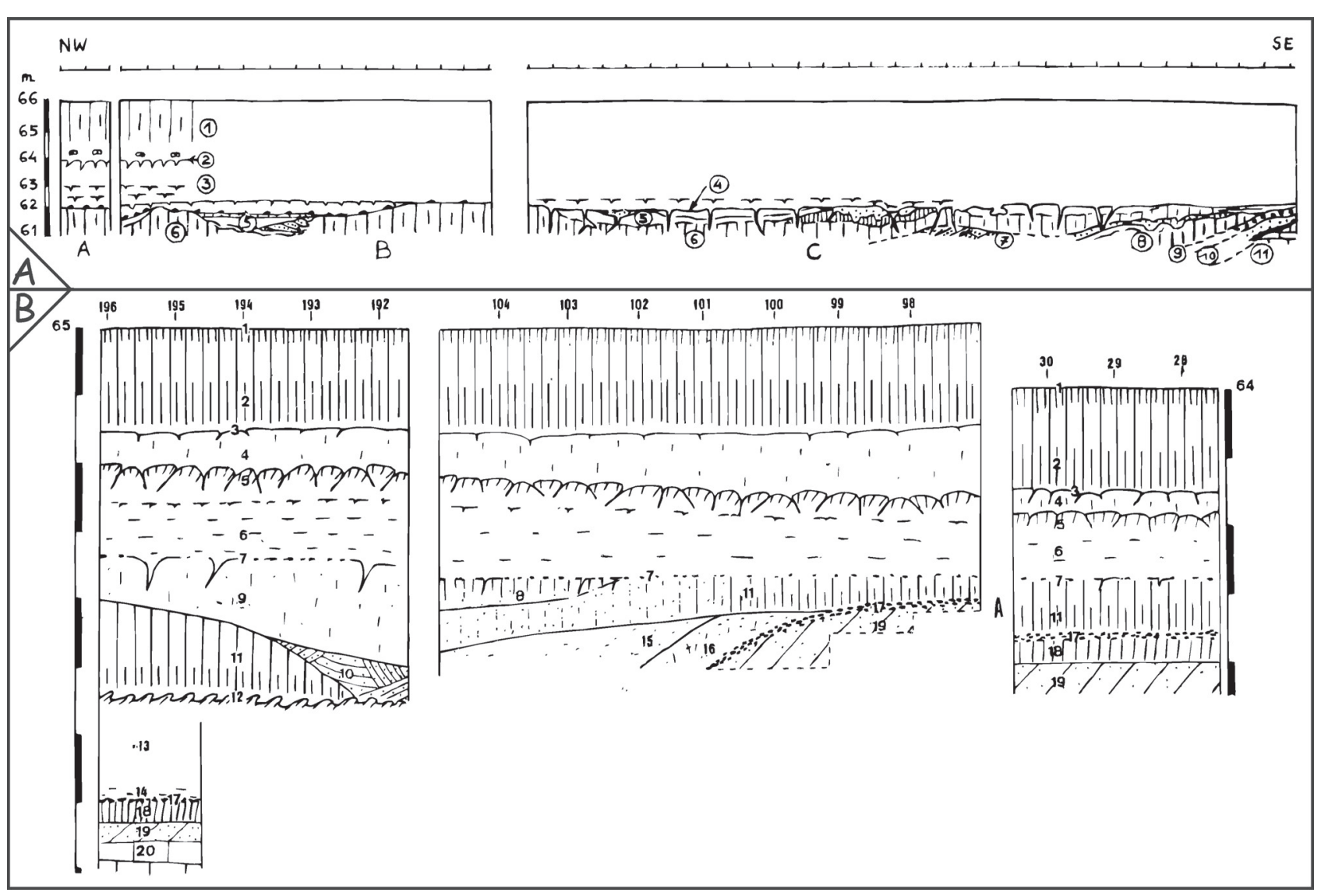

Fig. 2 - Coupe de la briqueterie de Vimy (localisation : $x=635 ; y=297,4 ; z=66$ NGF Lallemand)

A (d'après Sommé, 1975 ; figure 79) : Weichselien : 1/loss de couverture, 2/horizon cryoturbé (Kesselt), 3/formations limoneuses litées, 4/horizon de grandes fentes de gel, 5/sables et limons, avec localement sol de Warneton; Eemien et Pléistocène moyen : 6/paléosol hydromorphe (sol de Rocourt), 7/sables à structure entrecroisée, 8/limon et paléosol rougeâtre, 9/cailloutis de silex, 10/sable landénien remanié; 11/craie

B (d'après Sommé \& Tuffreau, 1976) : (extraits de la description et de l'interprétation chrono-stratigraphique) 1/horizon humifère, 2/horizon Bt, 3/niveau argileux gris-verdâtre, réseau de petites fentes, 4 /limon jaune-brunâtre, non calcaire ; concrétions calcaires, $5 /$ limon brunâtre légèrement argileux, localement cryoturbé, cailloux épars, réseau de petites fentes (Kesselt), 6/limon jaune-brunâtre, finement lité, nombreuses petites fentes de gel et cryoturbation en partie inférieure, 7/cailloutis de petits silex et ouverture de fentes de gel effilées (péjoration climatique du Pléniglaciaire inférieur), 8/limon brun-grisâtre, légèrement argileux, n'existe que localement (sol de Warneton), 9 et 10/ complexe de formations sableuses et sablo-limoneuses (début du Weichselien) : 9/limon jaunâtre, 10/lentilles sableuses à stratifications entrecroisées, 11/limon brunrougeâtre à brunâtre, compact, fendillé (sol interglaciaire eemien), 12/limon grisâtre, argileux, cryoturbé, réseau de fentes de gel, 13/limon doux brun-jaunâtre non lité, poupées et granules de craie, 14/limon doux, brun-jaunâtre, finement lité, poupées et granules de craie, 15/niveaux sablolimoneux jaune-verdâtre ou grisâtre, 16/limon argileux compact, 17/cailloutis de galets landéniens et silex 18/limon argileux, brun-grisâtre, compact 19/sable landénien altéré 20/craie sénonienne altérée

Fig. 2 - Vimy's brickyard section (location : $x=635 ; y=297,4 ; z=66$ NGF Lallemand survey)

A (according to Sommé, 1975, figure 79): Weichselien : 1/loess cover, 2/geliturbated layer (Kesselt), 3/laminated loamy formations, 4/deep frost cracks, 5/sands and silts, with Warneton soil in places ; Eemien and Middle Pleistocene : 6/hydromorphic palaeosoil (Rocourt soil), 7/cross bedded sands, 8/redish silt and palaeosoil, 9/flint gravel, 10/reworked landenien sand;11/chalk

B (according to Sommé \& Tuffreau, 1976): (description and chrono-stratigraphic interpretation extracts) 1/humic layer, 2/Bt layer, 3/greenish grey clay, frost cracks net, 4/brownish-yellow loam, non calcareous; calcareous concretions, 5/light clayey brownish loam, geliturbated in places, scattered gravels, frost-cracks net (Kesselt), 6/ laminated brownish-yellow loam, numerous little frost-cracks in the bottom part, 7/little flint gravels, thin and long frost-cracks opening (Lower pleniglacial climatic pejoration), 8/light clayey greyish-brown loam, only in places (Warneton soil), 9 et 10/ sandy loams and sands formations complex (Early Weichselian) : 9/yellowish loam, 10/cross-bedded sandy lens, 11/fissured, tight, reddish-brown to brownish loam, tight, fissured (eemian interglacial soil), 12/greyish loam, clayey, geliturbated, frost-cracks net, 13/non bedded yellowish-brown loam, chalk gravels and calcareous concretions, 14/ bedded yellowish-brown loam, chalk gravels and calcareous concretions, 15/grey or greenish-yellow loamy sand layers, 16/tight clayey loam, 17/landenian flint pebbles, 18/tight greyish-brown clayey loam, 19/weathered landenian sand, 20/weathered senonian chalk

\section{Unité 4 : Limon jaunâtre décalcifié sous l'unité 3}

De nombreux sondages montrent un front de décarbonatation plus bas que la base de l'horizon Bt. Le phénomène se développe au dépend de l'unité 5 sous un horizon Bt mince ou, avec une ampleur un peu plus importante, dans une légère dépression vers le bas du versant (SP6 où le front de décarbonatation dépasse $3,5 \mathrm{~m}$ de profondeur, transect 1 , fig. 3 ). Ce limon décalcifié peut garder la trace de réseaux de polygones ou de petits horizons de gley, mieux perçus dans l'unité 5.
Aucun autre phénomène macroscopique d'illuviation ou de lessivage associé n'a été perçu.

\section{Unité 5 : Loess calcaire homogène}

Un lœss clair, jaunâtre et calcaire couvre le versant exposé à l'est, avec épisodiquement des graviers de craie ou des horizons gleyifiés grisâtres qui sont souvent (mais non systématiquement) associés à un réseau de polygones. Jusqu'à trois horizons ont été distingués (SP 26 et 27). L'unité 5 est localement très épaisse et 


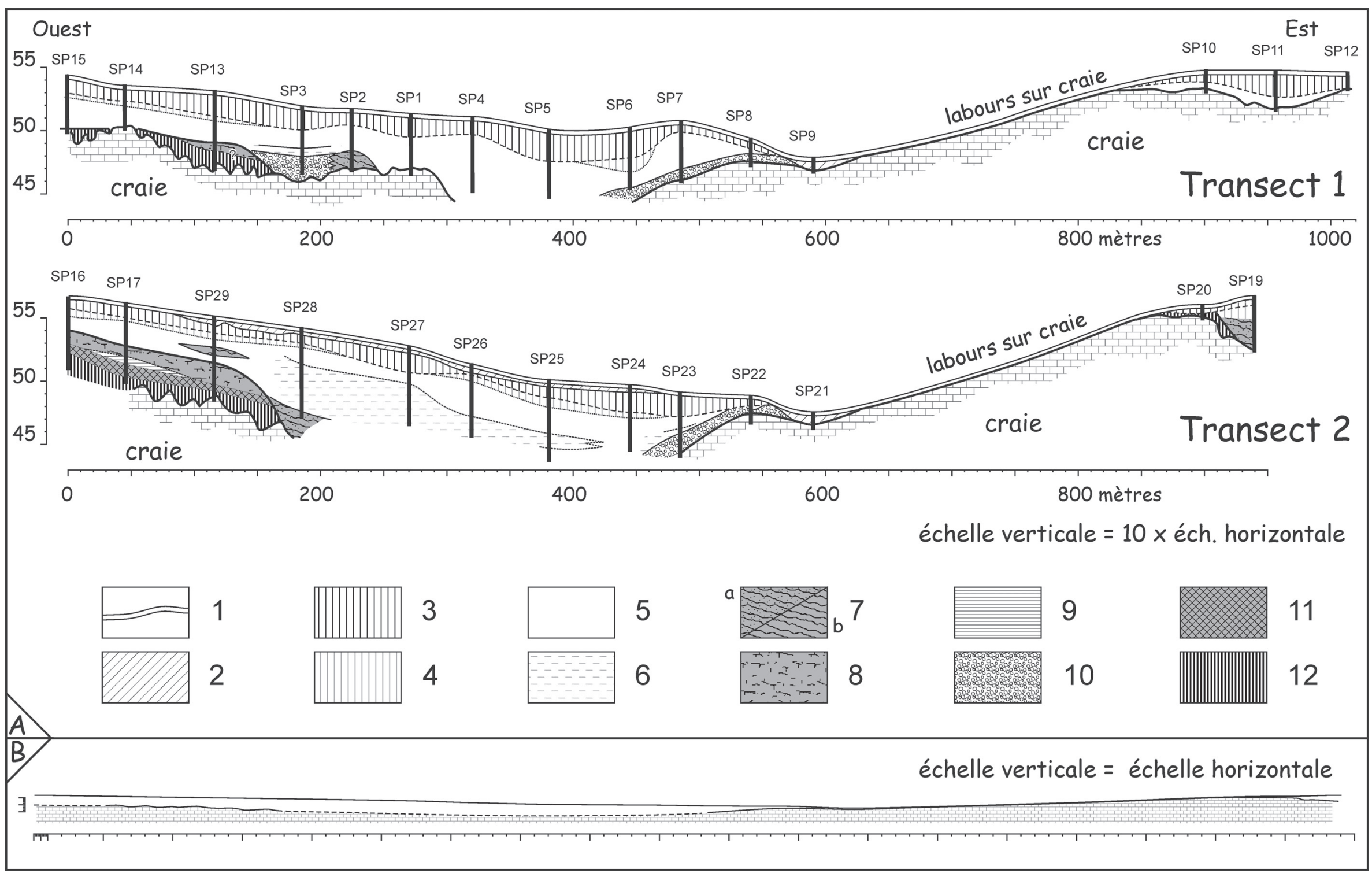

Fig. 3 - Transects du vallon sec

A/ transects détaillés. Unités stratigraphiques 1 à 12 : voir texte ; B/ transect 2 simplifié (Pléistocène et substrat crayeux)

Fig. 3 - Dry valley cross-sections

A/detailed cross-sections. Stratigraphic units 1 to 12 : see text; B/ simplified transect 2 (Pleistocene and chalky substratum) 
homogène. Le sondage le plus élevé (SP18, non utilisé pour la construction des transects) a traversé une forte épaisseur de lœss avec seulement un léger horizon gleyifié vers $6,5 \mathrm{~m}$ de profondeur. Seul le sondage SP1 a rencontré un horizon à langues (sol strié, coloration brune) vers $2,5 \mathrm{~m}$ de profondeur.

A l'échelle du transect, les graviers de craie inclus dans l'unité 5 sont plus abondants en sommet du versant ou en position d'interfluve, associés à des réseaux de petits polygones : sondages SP14 à 16. En outre, dans ces derniers, ils se situent en partie supérieure de l'unité.

\section{Unité 6 : Limon lossique calcaire lité}

De nombreux sondages montrent un lœss discrètement lité, en particulier sur le transect 2. Dans le sondage SP19, en position d'interfluve, il est enrichi en graviers de craie (6B sur la figure 3 ). Il est probable que l'unité 6 ait une extension plus importante mais que de mauvaises conditions d'observation (en particulier sur le transect 1) aient gêné sa perception (au profit de l'unité 5).

Unité 7 : Limons non calcaires en lits épais, postérieurs à 8

Quelques sondages situés vers le haut du versant exposé à l'est montrent une unité stratigraphique originale :

7A) Dans les sondages SP2 et SP28, il s'agit de lits limoneux épais (de l'ordre de $10 \mathrm{~cm}$ ), orangés, non calcaires, mais en alternance avec des lits très calcaires incluant des graviers et des petits blocs de craie, à peine émoussés.

7B) Dans une position topographique proche, le SP29 a rencontré des lits de limon non calcaire orangé. Le faciès est comparable à $7 \mathrm{~A}$ mais est dépourvu de tout passage calcaire.

\section{Unité 8 : Limon orangé non carbonaté et structuré}

Un limon orangé avec une structure aux agrégats légèrement arrondis est présent sur l'interfluve ouest (SP13, 16, 17, 29). Il est non calcaire ou très faiblement carbonaté (néoformation de carbonates provenant de l'unité 5 sus-jacente). Il est susceptible d'avoir une extension plus importante mais est difficile à identifier en l'absence de l'unité 11 qui le distingue nettement de l'unité 12 .

\section{Unité 9 : Limon non calcaire en lits fins, antérieur à 8}

Le SP17 a rencontré sous l'unité 8 et sur l'unité 11 , un limon finement lité brun orangé non calcaire. Par son faciès et par sa position stratigraphique, 9 se distingue nettement de l'unité 7.

\section{Unité 10 : Graviers de craie roulés}

Les sondages SP6 à 8, 22 et 23 ont rencontré un dépôt de gravier roulé, lité, meuble, avec un peu de matrice sableuse, directement sur la craie fragmentée. L'unité présente un pendage net inverse à la pente du versant et s'épaissit rapidement (plus de 1,5 m dans le SP23). L'unité 10 est directement scellée par les lœss de l'unité 5. Un dépôt similaire se trouve isolé dans le SP3, à une altitude comparable.

\section{Unité 11 : Horizons de sol fortement structurés}

La partie la plus élevée de l'interfluve ouest (au-dessus de $55 \mathrm{~m}$ ) présente une succession d'horizons gris sombres, gris clairs puis gris bruns, avec une structure lamellaire fine, bien marquée, à revêtement limoneux clair. Le complexe formé par ces horizons a été clairement observé dans les sondages SP16, SP17 et SP19.

Unité 12 : Limon argileux orangé, structuré, sous l'unité 11

En l'absence d'observations fines des coupes, la limite inférieure de l'unité 11 peut être difficile à établir et nous avons interprété comme unité 12 le passage à un sédiment plus compact, plus orangé, avec des agrégats plus massifs. Celle-ci a été observée sur l'interfluve ouest, recouvrant la craie à la topographie irrégulière (le contact est souligné par une zone peu épaisse d'argile brune avec manganèse).

Quand les horizons gris supérieurs de l'unité 11 sont absents du sondage, il est également difficile de séparer clairement en sondages les unités 10 et 12 (transect 1 ).

Enfin, nous associons à l'unité 12 un limon argileux rougeâtre (de coloration plus vive que l'horizon profond du sol actuel) avec quelques lits de sable et des galets de silex à cortex verdi rencontrés dans le sondage SP20, sur le versant opposé.

\section{3 - INTERPRÉTATION CHRONO- STRATIGRAPHIQUE ET COMMENTAIRES}

\section{1 - UNE SÉQUENCE LESSIQUE CARACTÉ- RISTIQUE DU PLÉISTOCÈNE SUPÉRIEUR EN POSITION D'INTERFLUVE}

La séquence limoneuse sur l'interfluve ouest correspond à un bilan sédimentaire fréquent pour le Pléistocène supérieur régional (Antoine, 2002; Antoine et al., 2003), avec du bas vers le haut (fig. 3 et 4) :

- le sol interglaciaire eemien (unité 12);

- le «sol gris forestier» développé sur colluvions (partie inférieure de l'unité 11) puis le «sol gris steppique » (partie supérieure de l'unité 11), attribuable au Début Glaciaire Weichselien ;

- une formation limoneuse litée (unité 9) attribuable au Pléniglaciaire inférieur ;

- le sol interstadiaire du Pléniglaciaire moyen (unité 10)

- le lœss lité (unité 6) ou homogène (unité 5) attribuable au Pléniglaciaire supérieur.

\section{2 - UNE IMPORTANTE ÉROSION ANTÉRIEURE AU LESS DU PLÉNIGLACIAIRE SUPÉRIEUR}

L'unité 7A a pour particularité d'être située à mi-versant, à proximité de la remontée du toit de la craie et des formations limoneuses non calcaires qui la scellent (unités 8, 9, 11 et 12). L'unité 7A est interprétée comme remaniement de ces sols, associés au remaniement du 


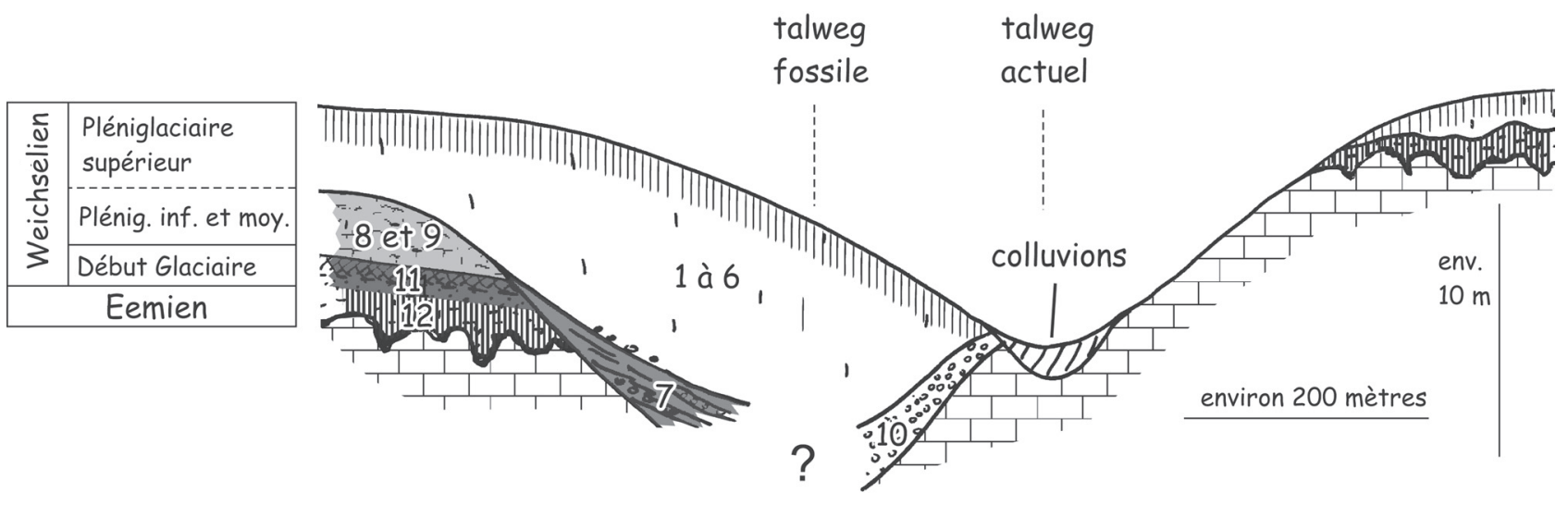

Fig. 4 - Transect simplifié et interprétation chrono-stratigraphique (légende dans le texte).

Fig. 4 - Simplified cross-section and chrono-stratigraphic interpretation (see text for legend).

substrat crayeux affleurant (probablement par solifluxion). Ce processus permet d'expliquer la calcimétrie contrastée de l'unité (alternance de limon non calcaire et de bandes de craie) et sa coloration rougeâtre (héritée des pédogenèses antérieures). Ce phénomène rappelle les observations épisodiques de remaniement en masse du sol eemien au sein du complexe de sol du Début Glaciaire (Hermies : Antoine et al., 1994). A Avion, même si le sol eemien a probablement été affecté, il n'a pas conservé ses caractéristiques structurales.

L'unité 7B a vraisemblablement une origine identique à celle de 7A, mais elle est située un peu plus haut et dans un secteur où la craie est masquée. Les remaniements limoneux n'ont pas été enrichis en éléments crayeux.

Directement scellée par les lœss du Pléniglacaire, l'unité 7 peut être attribuée à la phase d'érosion observée dans le bassin parisien, à la transition Pléniglaciaire moyen/supérieur (Antoine, 2002 ; Antoine et al., 2003 ; Locht et al., 2003). Mais contrairement à la Somme ou à Villiers-Adam (Locht et al., 2003), le dépôt n'est pas finement lité et n'est pas confiné à de grandes ravines descendant du versant. Toutefois la méthode d'observation ne permet pas d'exclure formellement l'existence de telles formes ni un éventuel diachronisme de l'unité 7 : il est possible que la même érosion se soit produite à la transition Début Glaciaire/Pléniglaciaire inférieur. D'ailleurs la coupe de la briqueterie montre clairement une érosion en ravines (fig. 2, n ${ }^{\circ}$ ) qui est attribuable au début du Pléniglaciaire (fig. 5).

\section{3 - L'ENREGISTREMENT D'ÉCOULEMENTS ANTÉRIEURS AU LCESS DU PLÉNIGLACIAIRE SUPÉRIEUR}

Sur le versant fossile opposé, le lœss a scellé une unité de graviers de craie roulés, avec une légère matrice sableuse (unité 10) qui repose directement sur la craie fragmentée. Nous l'interprétons comme un épandage fluviatile, témoin d'écoulements compétents, au moins épisodiques, dans le talweg fossile.
La création du réseau des talwegs aujourd'hui secs ou temporairement actifs dans les parties amonts des bassins versants est attribuée à des environnements périglaciaires favorisant l'écoulement, notamment grâce à la présence d'un pergélisol (Bravard \& Petit, 1997). La présence d'alluvions grossières dans ces talwegs est fréquente et des accumulations fluviatiles importantes, sous des dépôts éoliens, ont été maintes fois observées dans des bassins versants proches. Entre autres exemples :

- A Onnaing, dans le bassin de l'Escaut, des sables entrecroisés avec des rognons de silex se sont accumulés au débouché d'un vallon (Deschodt et al.,1998), sans connexion perçue avec le pédo-complexe du Début-glaciaire weichselien, bien développé sur le versant proche

- En bordure de la vallée de la Deûle, à Seclin et plus au nord à Lambersart, des alluvions - craie et sable à Seclin (Deschodt \& Boulen, inédit), sable et graviers d'argile à Lambersart (Deschodt, 1996) - incisent un limon gleyifié attribué Début-glaciaire. Dans les deux cas les formations grossières ont été recouvertes de lœss.

- Un peu plus au nord, à Poperinge (Belgique), Paepe et Vanhoorne (1967) ont observé des sables entrecroisés (« cross-bedded sands ») datés du début du «Pleniglacial B » (soit du Pléniglaciaire supérieur, Paepe \& Sommé, 1970). Ces premières alluvions sont recouvertes d'une couverture limoneuse lœssique qui montre une transition rapide avec toujours des dépôts alluviaux de sable entrecroisés. Cet ensemble, qui témoigne de la persistance d'un écoulement compétent pendant le dépôt de lœss, est recouvert par les sables de couverture de la fin du Pléniglaciaire supérieur et du Tardiglaciaire.

A Avion, l'unité 10 n'a pu être observée dans toute son extension latérale du fait de la puissance du lœss sus-jacent. Comme à Onnaing, le contact alluvions/lœss est brutal (sans remaniements ou altérations perceptibles) et les relations stratigraphiques entre alluvions et sols weichseliens ne sont pas établies (fig. 4). Le sondage SP3 (fig. 3) a rencontré l'unité 10 à l'ouest du transect 1 , dans une position isolée (fig. 3) mais à la même altitude que le sommet du gravier situé 


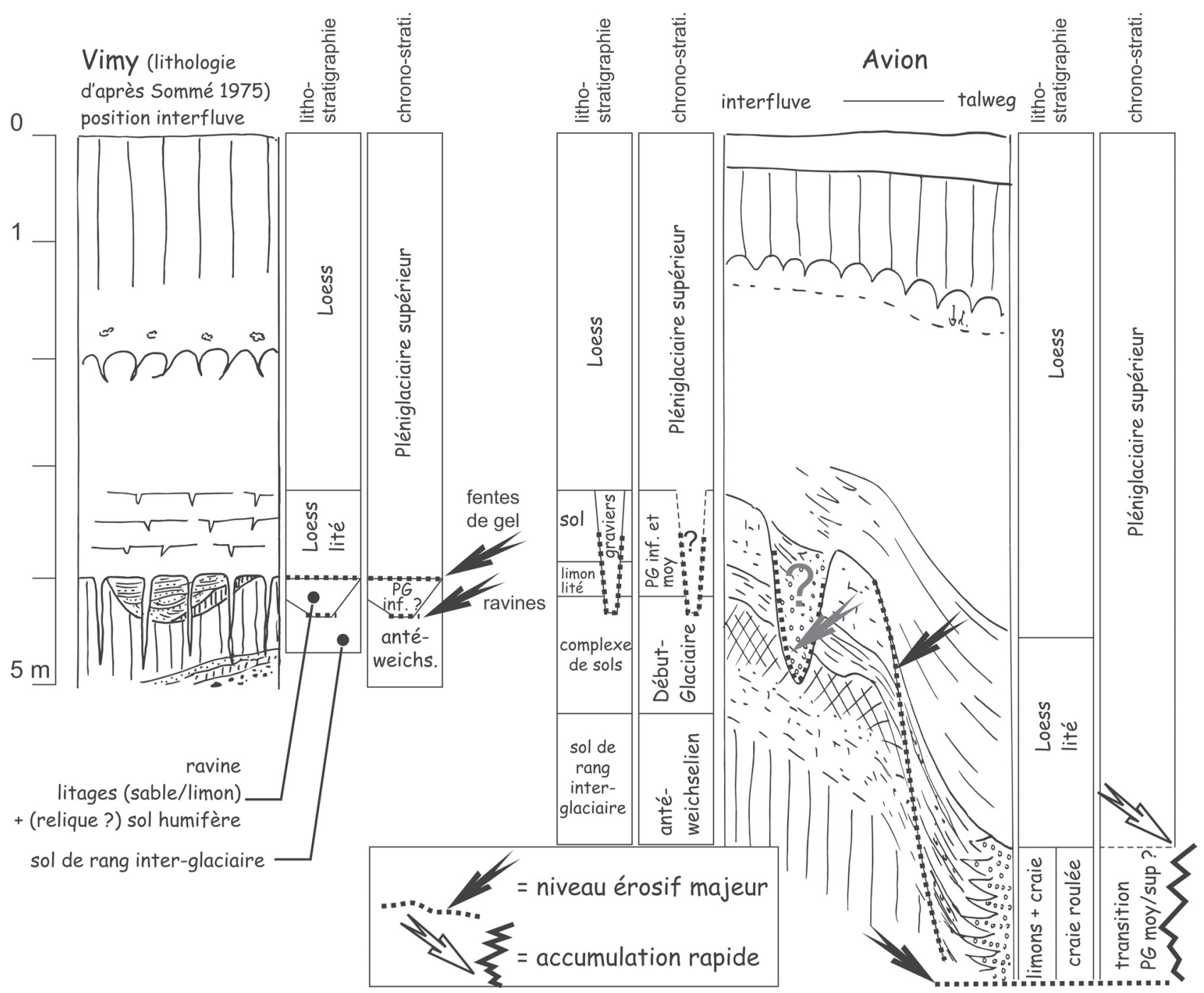

Fig. 5 - Comparaison Vimy/Avion et interprétation chrono-stratigraphique Fig. 5 - Vimy/Avion comparaison and chrono-stratigraphic interpretation

le plus à l'est (SP8). Nous l'interprétons comme un dépôt en ravine qui incise l'interfluve.

\section{4 - COMPARAISON AVEC LA COUPE DE VIMY ET ESSAI DE SYNTHÈSE STRATIGRAPHIQUE : DEUX NIVEAUX ÉROSIFS MAJEURS}

Un essai de synthèse est proposé sur la figure 5. La partie supérieure des séquences ne présente pas de difficulté de corrélation : composée de lœss et de lœss lité, elle peut être attribuée au Pléniglaciaire supérieur.

A Vimy, deux niveaux d'érosion sont perceptibles :

- une troncature avec fentes de gels à la base de la séquence lœssique,

- de petites ravines à remplissage de sable et limon avec relique d'un sol humifère.

Il est possible que le sol humifère (qui tapisse le fond de la ravine), soit le témoin d'un remaniement en masse, contemporain du développement de la ravine. Quoi qu'il en soit, le comblement lité de la ravine est postérieur au Début-Glaciaire. Il peut être attribué à la transition Début-Glaciaire/Pléniglaciaire ou au début du Pléniglaciaire inférieur. Le phénomène érosif et sa datation seraient alors très proches de ceux observés à Villiers-Adam (Locht et al., 2003). A Vimy, la présence de ravines en position sommitale d'un interfluve témoigne d'une phase érosive particulièrement intense.

Aucun équivalent strict n'est observé sur les transects d'Avion ou sous la séquence lœssique :

- en position d'interfluve et en sommet de versant, une séquence du Début-Glaciaire au Pléniglaciaire moyen est conservée sans érosion majeure perceptible (à l'exception peut-être d'un écoulement fluviatile en ravine soupçonné à travers le SP3) ;

- une formation litée est issue d'un remaniement du versant (unité 7),

- une formation fluviatile tapisse le talweg (unité 10).

Bien que nous ne possédions pas d'évidence de leur contact, nous supposons que les unités 7 (remaniements sur versant) et 10 (dépôts alluviaux dans le talweg) sont contemporains et issues majoritairement de la même phase de déstabilisation. Nous proposons de les associer à la phase de dégradation du pergélisol, à la transition Pléniglaciaire moyen / Pléniglaciaire supérieur, qui précède à Vimy le dépôt de la couverture lœssique sur le niveau érosif à fentes de gel (fig. 5 et 6). 


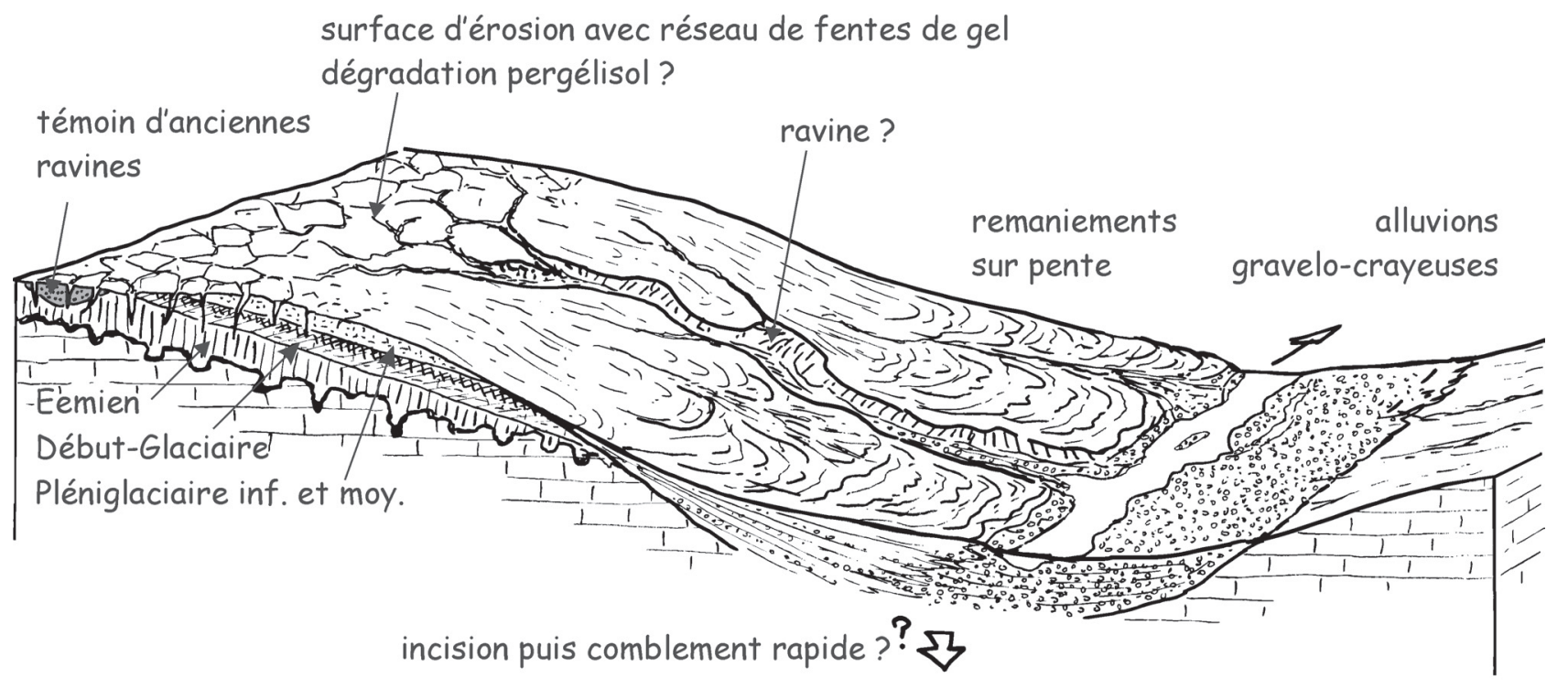

Fig. 6 - Hypothèse de fonctionnement du vallon à la transition Pléniglacaire moyen / supérieur ou au début du Pléniglaciare supérieur Fig. 6 - Valley activity at the Middle / Upper Pleniglacial or early Upper Pleniglacial hypothesis

\section{5 - RÉFLEXION SUR LA GENÈSE DU RÉSEAU DE TALWEGS}

Notre interprétation implique que l'essentiel des sédiments grossiers présents sous le lœess dans le fond du talweg fossile date de la transition Pléniglaciaire moyen / Pléniglaciaire supérieur. La présence sur l'interfluve de Vimy de petites ravines (à la transition Début-Glaciaire / Pléniglaciaire) indique que cette phase d'activité n'est pas unique dans le Weichselien et, qu' au moins à deux reprises, les talwegs aujourd' hui secs ont collecté un ruissellement important en provenance des interfluves. Ces phases d'érosion impliquent des conditions environnementales très particulières susceptibles de générer d'importants écoulements sur de faibles surfaces : climat relativement humide avec une faible couverture végétale, avec peut-être un pergélisol en cours de dégradation impliquant un fort ruissellement? La répétition de tels événements, bien que probablement courts, a du être déterminante dans la constitution du réseau de talwegs et, par l'introduction d'un important flux solide et liquide, ont probablement eu des répercussions importantes sur les systèmes fluviaux.

\section{6 - UN VALLON FOSSILISÉ PAR UN APPORT MASSIF DE LCESS AU PLÉNIGLACAIRE SUPÉRIEUR}

Les transects révèlent sans ambiguïté un dépôt massif de lœss sur le versant exposé à l'est (protégé des vents d'ouest). L'importance du phénomène est telle que le talweg antérieur au dépôt est remblayé (fig. 4) et que le talweg actuel résulte d'une légère incision directement dans la craie, avec un décalage notable vers l'est (environ 200 mètres) et vers le haut (dénivellation de 5 mètres ou plus).

La dissymétrie marquée de l'enregistrement sédimentaire (opposition versant au vent / sous le vent) se répercute mal sur le profil transversal des talwegs (la dissymétrie du vallon reste modérée). La sédimentation éolienne n'est pas contrebalancée par le recul du versant au vent. Ce phénomène est peut-être du à l'absence d'évacuation des débris cryoclastiques par des écoulements fluviatiles. Il se traduit par une tendance générale au remblaiement du vallon.

\section{6 - CONCLUSION}

1 - Malgré les discontinuités de sa couverture lœssique, la Gohelle est favorable à la conservation d'enregistrements quaternaires. Des sols du Début-glaciaire et du Pléniglaciaire weichselien sont présents sur les interfluves et le dépôt de lœss du Pléniglaciaire supérieur peut dépasser 7 mètres sur le versant protégé des vents d'ouest.

2 - Le vallon étudié présente un système morphosédimentaire original (vraisemblablement généralisable à une partie de la Gohelle) où l'apport massif de lœss n'est pas compensé par le recul du versant crayeux au vent. Il en résulte une tendance au remblaiement et à l'exhaussement du fond du vallon au cours du dernier cycle glaciaire. Le talweg actuel se retrouve décalé vers l'Est et perché sur le versant par rapport à l'incision maximum. La simplicité du réseau actuel découle en partie de l'enfouissement du réseau anté-weichselien sous les lœss pléniglaciaires.

3 - Des formations fluviatiles grossières sont conservées dans le talweg fossilisé par le lœss du Pléniglaciaire supérieur. Par ces caractéristiques morphosédimentaires, la Gohelle est favorable à la conservation des dépôts fluviatiles anciens dans les talwegs élémentaires et donc aux études sur les maxima hydrologiques qui ont pu affecter la partie amont des bassins versants au cours du dernier cycle glaciaire. Le dernier événement fluviatile important, lié à une érosion majeure sur le versant, semble pouvoir être attribué à la transition Pléniglaciaire moyen/supérieur. Il a 
peut-être été précédé d'un phénomène identique à la transition Début-Glaciaire / Pléniglaciaire. Aucun phénomène fluviatile de cette ampleur n'a marqué le talweg depuis le Pléniglaciaire supérieur.

\section{REMERCIEMENTS}

Merci à Jennifer Lantoine pour son aide sur le terrain. Merci à Jean Sommé et Pierre Antoine pour leurs remarques constructives. Merci à J.L. Monnier pour la relecture des traductions anglaises.

\section{RÉFÉRENCES BIBLIOGRAPHIQUES}

ANTOINE P., 2002 - Les lœss en France et dans le Nord-Ouest européen. Revue française de Géotechnique, 99, 3-21.

ANTOINE P., MUNAUT A.V., \& SOMMÉ J., 1994 - Réponse des environnements aux climats du Début Glaciaire weichsélien : données de la France du Nord-Ouest. Quaternaire, 5, 151-156.

ANTOINE P., AUGUSTE P., BAHAIN J.J., COUDRET P., DEPAEPE P., FAGNART J.P., FALGUÈ RES C., FONTUGNE M., FRECHEN M., HATTE C., LAMOTTE A., LAURENT M., LIMONDIN-LOZOUET N., LOCHT J.L., MERCIER N., MOIGNE A.M., MUNAUT A.V., PONEL P., \& ROUSSEAU D.D., 2003 - Paléoenvironnements pléistocènes et peuplements paléolithiques dans la Somme (nord de la France). Bulletin de la Société Préhistorique Française, 100, 5-28.

BRAVARD J.P., \& PETIT F., 1997 - Les Cours d'eau. Dynamique du système fluvial. Armand Colin, Masson, Paris, 222 p.

DESCHODT L., 1996 - Le Quaternaire de Lambersart «Les Conquérants ». Les Cahiers de la Préhistoire du Nord, 19, 135 p.
DESCHODT L., DJEMMALI N., DRWILA G., FÉRAY P., \& TEHEUX É., 1998 - Onnaing (59), usine Toyota. Rapport des sondages archéologiques profonds. AFAN, SRA Nord-Pas-de-Calais, 2 tomes, rapport non publié.

LEFÈVRE P., 2006 - Zone industrielle des 14. Diagnostic archéologique. INRAP, SRA Nord-Pas-de-Calais, rapport non publié.

LOCHT J.L., ANTOINE P., BAHAIN J.J., DRWILA G., RAYMOND. P., LIMONDIN-LOZOUET N., GAUTHIER A., DEBENHAM N., FRECHEN M., ROUSSEAU D.D., HATTE C., HAESAERTS P., \& METSDAGH H., 2003 - Le Gisement Paléolithique moyen et les séquences pléistocènes de Villiers-Adam (Val-d'Oise). Chronostratigraphie, environnement et implantations humaines. Gallia Préhistoire, 45, 1-111.

PAEPE R., \& SOMMÉ J., 1970 - Les lœss et la stratigraphie du Pléistocène récent dans le Nord de la France et en Belgique. Annales de la Société Géologique du Nord, 90-4, 191-201.

PAEPE R., \& VANHOORNE R., 1967 - The Stratigraphy and Palaeobotany of the Late Pleistocene in Belgium. Mémoire pour servir à l'explication des Cartes géologiques et minières de la Belgique, 8 , Ministère des affaires économiques, administration des mines, Service Géologique de Belgique, 96 p., 14 pl.

SOMMÉ J., 1975 - Les plaines du Nord de la France et leur bordure. Etude géomorphologique. Doctorat d'Etat, Université de Paris I. Edition 1977, Atelier reproduction des thèses, Lille, et Champion, Paris, 810 p., 185 fig.

SOMMÉ J., \& TUFFREAU A., 1976 - « Le Gisement acheuléen supérieur de Vimy (Pas-de-Calais)». In: G. Verron (dir.), NordOuest de la France (bassin de la Seine, bassin de la Somme et Nord). Livret-guide A 10 de l'excursion du $9^{e}$ congrès de l'Union Internationale des Sciences Préhistoriques et Protohistoriques, Nice, 191-194. 